\title{
Statistical modeling for risk assessment at sudden failures of construction equipment
}

\author{
Tatyana Rogovenko ${ }^{1}$, and Marina Zaitseva ${ }^{1, *}$ \\ ${ }^{1}$ Don State Technical University, Rostov-on-Don, Russia
}

\begin{abstract}
This paper applies the statistical modeling method to provide a quantitative assessment of risks emerging at sudden failures of construction equipment. The risk mathematical model takes into consideration the distribution parameters of operation, downtime and repair hours determined for a general population of the failed part finite volume. The proposed modeling algorithm is applied to assess risks at sudden dipper stick failures. The modeling results allow one to evaluate the tolerable period for the equipment use under the set risk levels.
\end{abstract}

\section{Introduction}

The relevance of research conduct in risk assessment for construction equipment is growing along with the enhancement of its security assessment methods [1-10].

Sudden failures represent one of the risk sources during construction equipment operation. In general, construction equipment is repairable, and in this connection, there is a vital necessity to assess risks with the account of the operation (running) influence and time as well as the recovery time. The multi-parameter Weibull model flexibility enables one to use it for approximation of the experimental data obtained in relation to recovery and running intervals including the small data selections [11]. The transition from the Weibull distribution parameters assessment for small data selections towards the general population finite volume (GPFV) parameters may be performed by means of the well-proven and successfully applied FISP method $[4,12]$.

\section{Statement of Problem}

The quantitative risk assessment for the construction equipment $t$ operation time can be calculated as a product of an undesirable event occurrence, i.e., a sudden failure, $P(t)$, and the consequences of this event in the form of damage $D(t)$ in compliance with the standard $[1,13]$ :

$$
R(t)=P(t) D(t) .
$$

Hence, new tasks arise: assessment of the operation time, downtime and repair time distribution parameters as well as determination of the damage caused by such failure for each of the time intervals. Solution of the mentioned problems requires application of the probability and statistics methods to assess distribution parameters for a data selection with a subsequent transition to the GPFV of the parts the failure of which is considered to be an undesirable event $[4,12]$.

The quantitative risk assessment mathematical model at the sudden failure can be represented in a certain way. Let us introduce the following notation: $n$ - failure number, $m$ - time interval number identified during the risk assessment process (in what follows $m=3$ ), $C_{R i}-$ repair costs after the $i$ failure, $t_{i j}-$ length of the $j$ time interval after the $i$ failure $(i=0,1, \ldots, n ; j=1,2, \ldots, m)$ (Fig. 1).

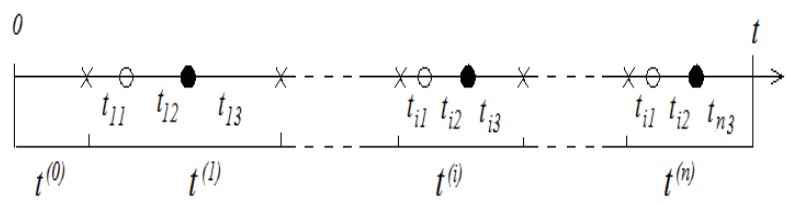

Fig. 1. Intervals tij on the time scale

Each of the $t_{i j}$ time intervals has its own probability distribution $F_{j}\left(t_{i j}\right)$ and the damage function $D_{j}\left(t_{i j}\right)$.

The operation time to assess risks is define using the formula

$$
t=t^{(0)}+\sum_{i=1}^{n} \sum_{j=1}^{m} t_{i j}
$$

The repair costs $C_{R i}$ can be determined in compliance with the procedure described in [14].

Obviously, for the notation introduced: $t_{0 I}=t^{(0)}-$ running time until the first failure, $t_{02}=0, t_{03}=0, C_{R 0}=0$.

The risks from sudden failures $n$ occurrence for the time $t$ including the $m$ time intervals may be calculated applying the following formula:

\footnotetext{
*orresponding author: marincha1@rambler.ru
} 


$$
R(t)=\sum_{i=l}^{n}\left(C_{R i}+\sum_{j=l}^{m} F_{j}\left(t_{i j}\right) D_{j}\left(t_{i j}\right)\right) .
$$

\section{Distribution parameters assessment}

The three-parameter Weibull distribution was chosen as the probability distribution for each of the $t_{i j}$ time intervals (operation time, downtime and repair time):

$$
F(t)=1-\exp \left(-\left(\frac{t-c_{S}}{a_{S}}\right)^{b_{S}}\right)
$$

where $a_{S}, b_{S}, c_{S}$ are parameters of the scale, form and location respectively, determined by the maximum likelihood method for the small volume data selection $n_{S}$ $=5-15$.

The FISP method can be helpful for the transition from the distribution parameters assessment over the $a_{S}, b_{S}, c_{S}$ small data selections towards the assessment of $a_{G}, b_{G}, c_{G}$ for the failed parts GPFV [12].

The FISP method is essentially about sequential simulation of finite intermediate subpopulations (FISP) $N_{T}=10^{2}-10^{4}$ using small samples of size $n_{S}=5-15$, which is followed by modeling the finite general population (FGP) $N_{G}=10^{4}-10^{6}$ using the correlative dependencies obtained in [15].

For such simulation, FISPs are determined by the number of additional values of random variable $m_{a d}$ based on the distribution law and depending on the extent of order statistics $R_{i i+1}=x_{i+1}-x_{i}$ as well as their distribution law:

$$
m_{a d}=\sum_{i=0}^{n_{S}}\left(n_{a d_{i}}+\Delta_{i}\right)
$$

where the components are calculated using the formula:

$$
\begin{gathered}
n_{a d_{0}}=\left[\frac{F^{*} \cdot\left(N_{T}-n_{S}-2\right)}{\left(x_{1}-A\right) \cdot \sum_{j=1}^{n_{S}-1}\left(\left(F\left(R_{j_{j+1}}\right)+F^{*}+F^{* *}\right) / R_{j_{j+1}}\right)}\right] \\
n_{a d_{i}}=\left[\frac{F\left(R_{i i+1}\right) \cdot\left(N_{T}-n_{S}-2\right)}{R_{i i+1} \cdot \sum_{j=1}^{n_{S}-1}\left(\left(F\left(R_{j_{j+1}}\right)+F^{*}+F^{* *}\right) / R_{j_{j+1}}\right)}\right]
\end{gathered}
$$

for $i=1, \ldots, n_{S^{-1}}$,

$$
\begin{gathered}
n_{a d_{n S}}=\left[\frac{F^{* *}\left(N_{T}-n_{S}-2\right)}{\left(B-x_{n_{S}}\right) \cdot\left(\sum_{j=1}^{n_{S}-1}\left(\left(F\left(R_{j_{j+1}}\right)+F^{*}+F^{* *}\right) / R_{j_{j+1}}\right)\right.}\right] \\
F^{*}=\min _{1 \leq i \leq n_{S}-1}\left\{\frac{F\left(R_{i_{i+1}}\right) \cdot\left(x_{1}-A\right)}{R_{i_{i+1}}}\right\} \\
F^{* *}=\max _{1 \leq i \leq n_{S}-1}\left\{\frac{F\left(R_{i_{i+1}}\right) \cdot\left(B-x_{n_{S}}\right)}{R_{i_{i+1}}}\right\}
\end{gathered}
$$

where $F\left(R_{i i+1}\right)$ - distribution of order statistics:

$$
F\left(R_{i i+1}\right)=\frac{n_{S} !}{(i-1) !\left(n_{S}-i+1\right) !} \times
$$

$$
\begin{aligned}
& \int_{0}^{R_{i i+l} C_{S}} \int_{A}^{-R r s} P^{i-1}(x) p(x) p\left(x+R_{i i+1}\right) \times \\
& \quad \times\left[1-P\left(x+R_{i i+1}\right)\right]^{n S^{-i+1}} d x d R,
\end{aligned}
$$

$P(x), p(x)$ are the probability function and density, $A$ and $B$ are the limits of the simulated range.

The value of $\Delta_{\mathrm{i}}$ is determined by successive approximations so that $N_{T}=n_{s}+m_{a d}+2$. If the order statistics are equal, the measurement error of the random variable is assumed as the extent value for simulating the additional values.

When debugging the FISP method, the homogeneity of the source population and the FISP was evaluated using the Wilcoxon test; the Smirnov-Kolmogorov test was used to verify the hypothesis that the distribution functions of the general populations the samples were taken from coincide in the entire domain. It was evaluated the share of FISPs $N_{W}=69-75 \%$. that are Wilcoxonhomogeneous to the initial population for a significance of $\alpha=0.01$; the share of FISPs $N_{K}=75-89 \%$. that share the general population with the source population (based on the Smirnov-Kolmogorov test for a significance of $\alpha=0.01 ; 0.001$.

\section{Risk modeling}

The statistical risk $R(t)$ modeling is intended for determining of the construction equipment operation critical time $t^{*}$ after which the risks exceed the set $R_{p}$ level.

In compliance with the preliminarily established laws for distribution of the operation time, downtime and repair time $F_{j}\left(t_{i j}\right) \quad(j=1,2, \ldots, m)$, risk modeling shall be repeated until the subsequent $R^{*}(t)$ value exceeds the preset level. The calculations may be repeated multiple times to determine the confidence bounds for the $t^{*}$ critical time assessment. Figure 2 shows the aggregate algorithm for risk modeling.

\section{Application}

The FISP simulation and quantitative risk assessment algorithm was implemented as a part of the MRM software suit developed by the Motor Vehicles, Construction and Road Machines Department, Don State Technical University, to study the problems of machine reliability and risk analysis.

The algorithm is applied to assess risks at sudden fatigue failures of the crane-type excavator EK-14 dipper stick.

The distribution parameters $F_{j}\left(t_{i j}\right)$ basic data after the FISP method [15] application for risk modeling are given in the Table 1 below. 
The $D_{j}\left(t_{i j}\right)$ damage functions are taken as being linearly dependent on time with the coefficients equal to the hourly cost of the excavator performance.

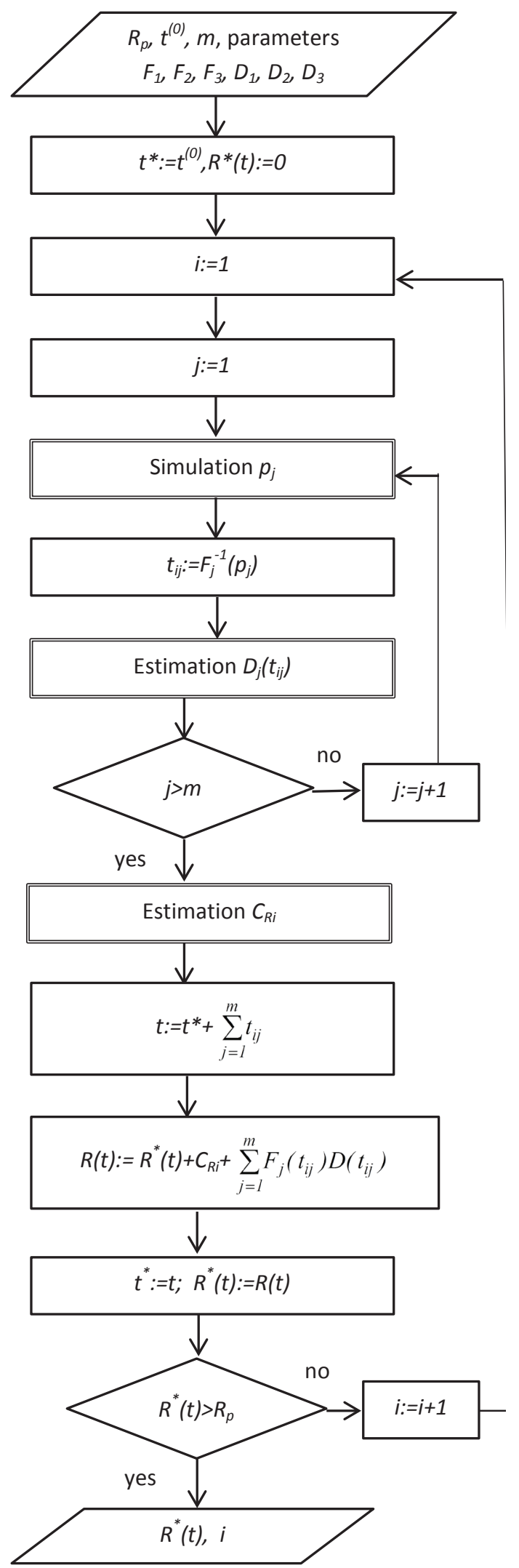

Figures 3-5 present the time interval distribution functions for selections and GPFV.

Figure 6 demonstrates one of the options of the $R(t)$ function modeling by means of accrual.

The multiply repeated $R(t)$ modeling allowed for determination of the variability interval for the operation time $t^{*}$ critical values under different $R_{p}$ risk levels reduced to the $C_{E}$. excavator cost. The results are given in the Table 2 .

Table 1. The initial data for modelling

\begin{tabular}{|c|c|c|c|}
\hline $\boldsymbol{t}_{\boldsymbol{i j}}$ & $\boldsymbol{a}_{\boldsymbol{G}}$ & $\boldsymbol{b}_{\boldsymbol{G}}$ & $\boldsymbol{c}_{\boldsymbol{G}}$ \\
\hline$t_{i 1}$ & 1850 & 2.1 & 16550 \\
\hline$t_{i 2}$ & 10 & 2 & 1.5 \\
\hline$t_{i 3}$ & 116 & 1.35 & 5.7 \\
\hline
\end{tabular}

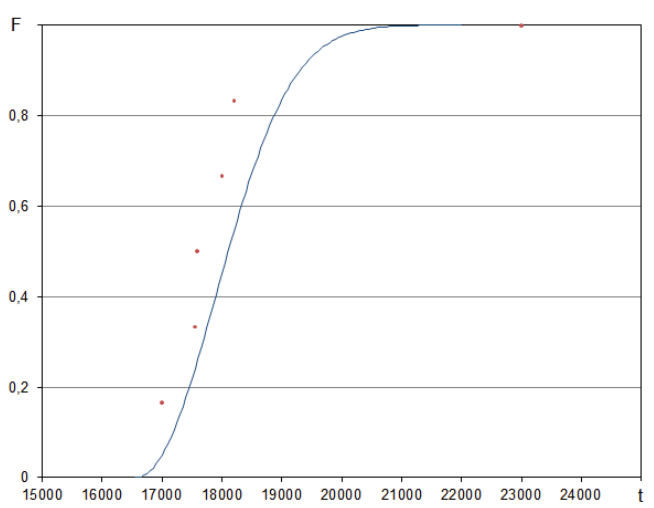

Fig. 3. Dipper stick operation time distribution

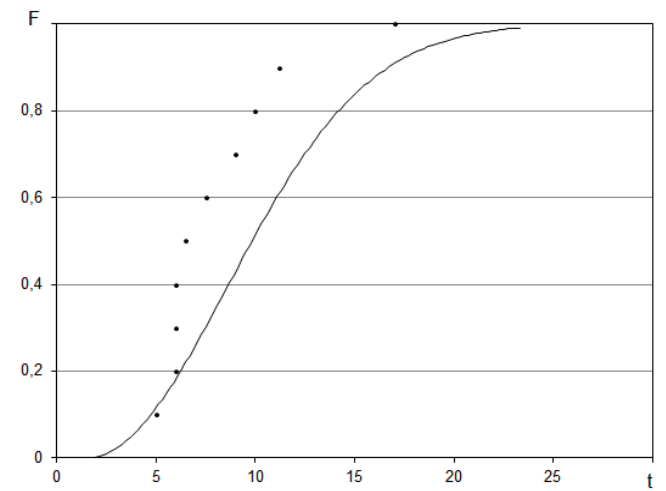

Fig. 4. Downtime distribution after a dipper stick sudden failure

Fig. 2.Risk modeling algorithm 


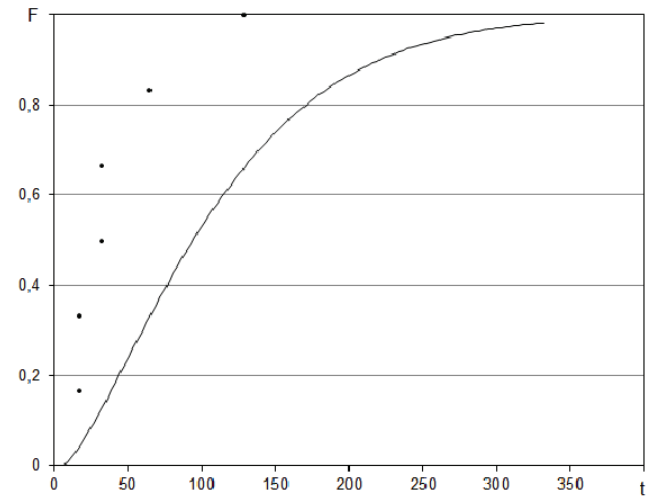

Fig. 5. Dipper stick repair time distribution

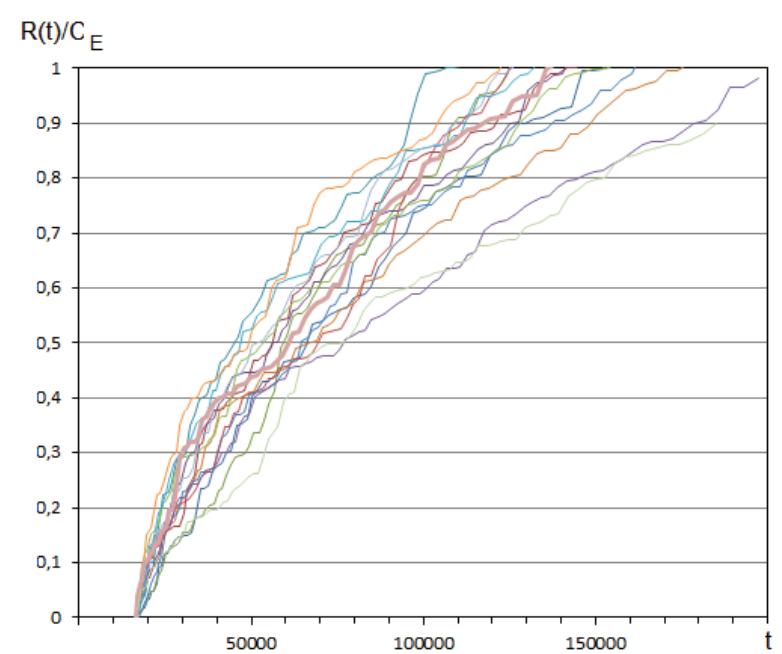

Fig. 6.Accumulated risks related to excavator cost

Table 2. Limits of the $t^{*}$ critical values

\begin{tabular}{|c|c|c|c|}
\hline$R_{p} / C_{E}$ & $t^{*}$ min & $\begin{array}{c}\text { the aver- } \\
\text { age value } \\
t^{*}\end{array}$ & $t^{*}$ max \\
\hline 0.1 & 18355 & 21053 & 24222 \\
\hline 0.2 & 21819 & 27760 & 36764 \\
\hline 0.5 & 44762 & 59976 & 77495 \\
\hline
\end{tabular}

\section{Conclusions}

Therefore, the proposed risk quantitative assessment mathematical model takes into account distribution parameters for the operation time, downtime and repair time for the failed parts GPFV at sudden failures of construction equipment. Risk statistical modeling allows taking a decision in terms of the further equipment use practicability and develop measures to mitigate sudden failure risks.

\section{References}

1. Handbook of risk theory. S. Roeser, R. Hillerbrand, P. Sandin,M. Peterson(Springer, 2012)

2. S.N. Goncharenko, E.V. Dement'eva. Min. Inf. \& Anal. Bul. (GIAB), 12, 95-104 (2010)

3. V.E. Kas'yanov, T.N. Rogovenko, Rus. Engin. Res., 6 (1999)

4. V. E. Kas'yanov, T.N. Rogovenko, M M Zaitseva, Bul. of Rost. St. Trans. Un., 1, 16-20 (2010)

5. B. Y. Renaulta, J. N. Agumba, MATEC Web Conf. (IBCC 2016) 66, 00008 (2016)

6. R. Stasiak-Betlejewsk, A. Czajkowska, MATEC Web Conf.( CoSME'16) 94, 04011 (2017)

7. V. E. Kas'yanov, L.P. Schulkin,ScienceRev., 10-2, 437-440 (2014)

8. V. E. Kas'yanov, L.P. Schulkin, ScienceRev., 10-3, 671-674 (2014).

9. V. E. Kas'yanov, L.P. Schulkin, ScienceRev., 11-3, 782-784 (2014)

10. D.Z. Evseev, S.N. Fil,ScienceRev., 11-2, 482-484 V. E. Kas'yanov, T.N. Rogovenko, M M Zaitseva, Bul. of the Don St. Tech. Un., 1, 91-94 (2010).

11. H. Rinne, The Weibull Distribution (2008)

12. M. M. Zaitseva, Obespecheniye zadannogo ustalostnogo pesursa detaley odnokovshovogo excavatora s ispolzovaniem malyh viborok (2010)

13. ISO/IEC 31010:2009 Risk management - Risk assessment techniques (IDT)

14. T.N. Rogovenko, M M Zaitseva, Eng. Jour. Don, 4, (2016)

15. V. E. Kas'yanov, T.N. Rogovenko, I. V. Topilin, Rus. Eng. Res., 3, 9-11 (2003) 